Research Paper

20214436

\title{
Development of Evaluating Methods for Passenger's Motion Sickness in Real Driving Environment
}

\author{
Shizuka Bando ${ }^{1)}$ Yuri Shiogai ${ }^{1)}$ Akinari Hirao ${ }^{1)}$ \\ 1) Nissan Motor Co., Ltd \\ 560-2, Okatsukoku, Atsugi-shi, Kanagawa 243-0192, Japan (E-mail: bando-s@mail.nissan.co.jp)
}

Received on February 19, 2021

\begin{abstract}
With the commercialization of autonomous driving technology and diversification of how passengers spend their time, it is concerned that a mismatch between somatosensory and visual information induces motion sickness. However, no effective quantitative method using physiological indices in driving conditions of a vehicle has been found out. The objective of this study is to develop a robust method for quantitative evaluation in real driving environment. As a result of inducing motion sickness of a back passenger by driving a vehicle on a test course, changes of forehead humidity, which reflects the amount of sweating, increased significantly. The result indicates forehead humidity is an effective index for quantifying motion sickness in real driving environment.
\end{abstract}

KEY WORDS: motion sickness, forehead humidity / Human Engineering, Car Sickness, Bioinstrumentation [C2]

\section{Introduction}

Autonomous driving technologies have been developed actively in recent years, with the Japanese government aiming to achieve fully automated driving on highways by $2025^{(1)}$. Under fully autonomous operation, on-board systems perform all dynamic driving tasks, eliminating the need for driver input. This will diversify how occupants spend their time inside vehicles including multitasking through devices such as laptops and tablets ${ }^{(2)}$. There are accordingly concerns that the lack of visual driving information and discrepancies between visual, vestibular, and somatosensory information may induce motion sickness (MS) ${ }^{(3-6)}$.

ISO 2631-1 ${ }^{\text {(7) }}$ defines Motion Sickness Dose Value (MSDV) as an index for evaluating MS based on the vibration level of acceleration and exposure time. MSDV assumes a single vibration frequency as a parameter, which thereby makes it difficult to apply to vehicles' running conditions containing various frequencies. In addition, not only whole body vibrations but also the discrepancies between visual, vestibular, and somatosensory information need to be taken into account for MS-inducing factors.

Symptoms of MS include nausea, vomiting, pallor, and cold sweat ${ }^{(8)}$. These are primarily symptoms of the autonomic nervous system, and hence researchers have evaluated various autonomic nervous system activity indicators such as heart rate variability (HRV), blood pressure, respiratory rate, electrodermal activity (EDA), blood flow, and skin temperature ${ }^{(9-14)}$. However, there are major individual differences in physiological responses at the onset of mild-to-moderate MS, and a consistent physiological evaluation index has not been established yet. Furthermore, no evaluation method in real driving conditions has been found out because most of the research has been conducted with simulators in the laboratory, where it is easier to control experimental environment.

The objective of this study is to develop a quantitative evaluation method for mild-to-moderate MS, the method that is effective in real driving environment. It is desirable for an evaluated index to work in mild-to-moderate MS and be specific response of MS, and the measurement of the index requires the simplicity of measurement procedure and robustness against vehicle noise.

This paper focuses on sweating, which is a specific response to MS and appear in mild-to-moderate MS. The authors evaluated the humidity above the forehead (HUM), which is easier to measure, in addition to EDA, which has been conventionally used to evaluate sweating. In order to evaluate automatic nervous system responses, the authors also measured cardiovascular indices such as HRV and forehead skin temperature (FHT).

Graybiel's test ${ }^{(15)}$ and the Motion Sickness Assessment Questionnaire (MSAQ) ${ }^{(16)}$ have often been used as subjective assessments of MS. These are based on subjective symptoms and vomiting, and not suitable for this study because no clear subjective symptoms may appear in mild-to-moderate MS. For this reason, the authors used a visual analog scale (VAS) to evaluate the degree of discomfort associated with MS.

In this paper, responses of these physiological indices to different intencities of MS were evaluated. The different intencities of MS were induced by video viewing tasks on a monitor in two position in real driving environment.

\section{Method}

\subsection{Measurement Environment}

The measurement system consisted of a vehicle instrumentation system for vehicle behavior indices, a bioinstrumentation system for physiological indices, and a logging system.

\subsubsection{Vehicle instrumentation system}

The vehicle instrumentation system consisted of (1) a controller area network (CAN) signal acquisition device (an in-house 


\section{Shizuka Bando et al / International Journal of Automotive Engineering}

Vol.12, No.2(2021)

product) for vehicle speed and accelerator opening, (2) an accelerometer (AU7684N1x00; Tamagawa Seiki) for vehicle acceleration, and (3) a thermometer (thermocouple wire and thermocouple connector; Ninomiya Electric Wire Co., Ltd. and Omega Engineering, respectively) for cabin temperature.

(1) The CAN signal acquisition device was connected to an electronic control unit of a vehicle through a harness. (2) The accelerometer was attached to the center console. (3) The thermometer was placed on the seatback of the left-side of rear seat where a participant was seated.

\subsubsection{Bioinstrumentation system}

The bioinstrumentation system consisted of (4) an accelerometer (AU7684N1x00; Tamagawa Seiki) for head motion, (5) an EDA meter (SPN01; Skinos Co., Ltd.) for skin potential level (SPL), (6) a hygrometer (SHT35 flexible type; Sensirion AG) for HUM, (7) a thermometer (thermocouple wire and thermocouple connector; Ninomiya Electric Wire Co., Ltd. and Omega Engineering, respectively) for FHT, and (8) an electrocardiogram (ECG) (Mini Wave; Cometa) for HRV .

(4) The accelerometer was attached to the top of the inner cap of the helmet which a participant wore. (5) Reference, positive, and negative electrodes of the EDA meter were attached to the right clavicle, Fp1 and Fp2 of the international 10-20 method, respectively. (6) The hygrometer was placed just above the center of the forehead. (7) The thermometer was placed on just under the hygrometer. (8) Electrodes of the ECG were attached to a participant according to modified lead II.

\subsubsection{Logging system}

The (1) to (7) data time series were recorded in a logger (an inhouse product) at the sampling frequency of $200 \mathrm{~Hz}$. The (8) data time series was recorder in a PC (Pavilion; HP) at the sampling frequency of $2000 \mathrm{~Hz}$.

The VAS is the subjective intensity determined by checking a position on a segment line with a pair of opposite adjectives written at both ends. Measurement items were "discomfort" associated with MS and "drowsiness". The adjectives were "no discomfort" "most discomfort" and "no drowsiness" - "most drowsiness".

\subsection{Measurement Procedures}

Experiments were conducted on a test course using a minivan vehicle. The test course was a circuit of approximately $700 \mathrm{~m}$, and it took approximately 60 seconds to complete one lap around the test course. A driver, a participant, and an experimenter sat in a driver seat, a left-side of rear seat, and a right-side of rear seat, respectively.

Figure 1 shows the experimental protocol. The experiment consisted a 60-second rest period (Rest), a 20-second question period $(\mathrm{Q})$, and a task period $(\mathrm{T})$, during which the vehicle drove one lap of the test course.

A driver stopped the vehicle on the side of the test course during Rest and $\mathrm{Q}$ periods. In $\mathrm{T}$ period, a driver made one lap, which contained four times of acceleration and deceleration from $20 \mathrm{~km} / \mathrm{h}$ to $50 \mathrm{~km} / \mathrm{h}$ and $50 \mathrm{~km} / \mathrm{h}$ to $20 \mathrm{~km} / \mathrm{h}$ for inducing MS. The acceleration and deceleration started and ended at the designated points on the course. An experienced driver made driving operations to ensure the repeatability of vehicle behavior indices of each lap.

The participant sat in the left-side of rear seat in a resting state with eyes closed during Rest period. In $\mathrm{Q}$ period, a participant checked the subjective intensity of discomfort and drowsiness on the VAS. In T period, a participant conducted a video viewing task

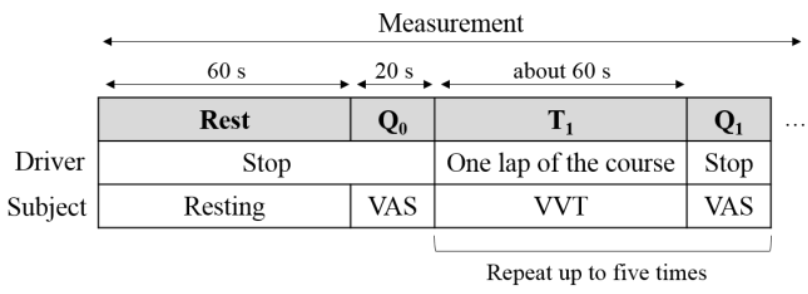

Fig. 1 Experimental protocol.

(VVT) to control participant's eyes. Under the VVT, the participant counts a number of events in the video which was presented on a monitor. In order to induce different intensities of discomfort associated with MS, an experimenter installed the monitor in two positions: the lower seatback of the front passenger seat (lower position; approximatry $0.6 \mathrm{~m}$ from cabin floor to the centor of monitor , 11 inch monitor) and the center of the roof (upper position; approximatry $0.1 \mathrm{~m}$ from cabin ceil to the centor of monitor, 11 inch monitor).

An experimenter asked a participant whether or not to continue the experiment at the $\mathrm{Q}$ period of each lap, stopped it if the participant wanted or at any time when the participant asked, and set $\mathrm{Q}$ period in the end of a trial. The maximum number of laps in one monitor-position was set to five.

The above experiment with a monitor on the lower or upper position was defined as one trial. All participants participated in two trials on different days, and performed VVTs under the same monitor position during one trial. The order of the two monitor position was decided in advance for each participant to eliminate the order effect.

The participants were 20 men and women in their 20 s to 50 s. An experimenter fully informed them about the experiments and the objective of this study prior to participation, and got informed consents from all participants. The participats were asked them to get sufficient sleep the day before, and prohibited them from eating, smoking, caffeine intake, and excessive exercise for one hour before the experiment. The experiments were carried out during the day to minimize the effects of circadian rhythm.

Nissan's Human Participants Research Ethics Committee approved this study.

\subsection{Evaluation Method}

The number of the total lap differed according to the degree of discomfort between the lower position and the upper position. The analysis target section was from Rest period to $\mathrm{n}$-th $\mathrm{T}$ period $\left(\mathrm{T}_{n}\right)$, where $\mathrm{n}$ was the minimum lap number of the two positions for a comparison between them, and SPL, HUM, and FHT were analyzed from Rest period to up to 20 seconds after $T_{n}$ period finished because of the 20-second latency of the physiological responses.

Since the main stimuli to a participant from the vehicle was the longitudinal stimuli associated with acceleration and deceleration, a longitudinal vehicle acceleration and head acceleration were analyzed. A low pass filter (Butterworth filter, cut-off frequency was set $3 \mathrm{~Hz}$, the frequency above which is not related to MS according to McCauley et. al. ${ }^{(17)}$ ) was applied to the acceleration, and jerk, time-differentiated acceleration, was calculated for vehicle and head in each $\mathrm{T}$ period. The evaluation indices of longitudinal vehicle acceleration and head acceleration were defined as a mean accumulated jerk ( $\overline{\text { Jerk }}$ ) per T period. 


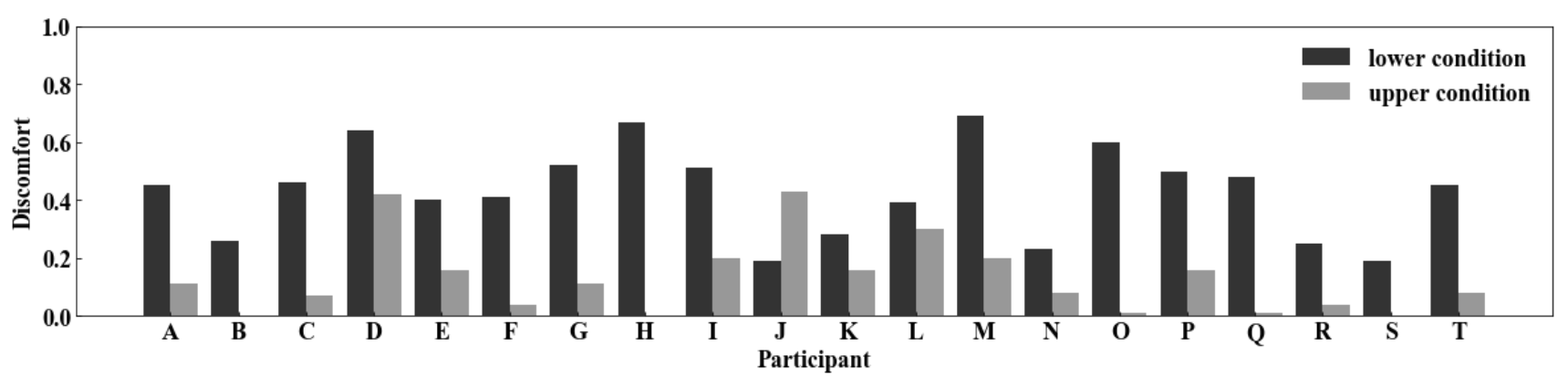

Fig. 2 Change amount of discomfort of each participant.
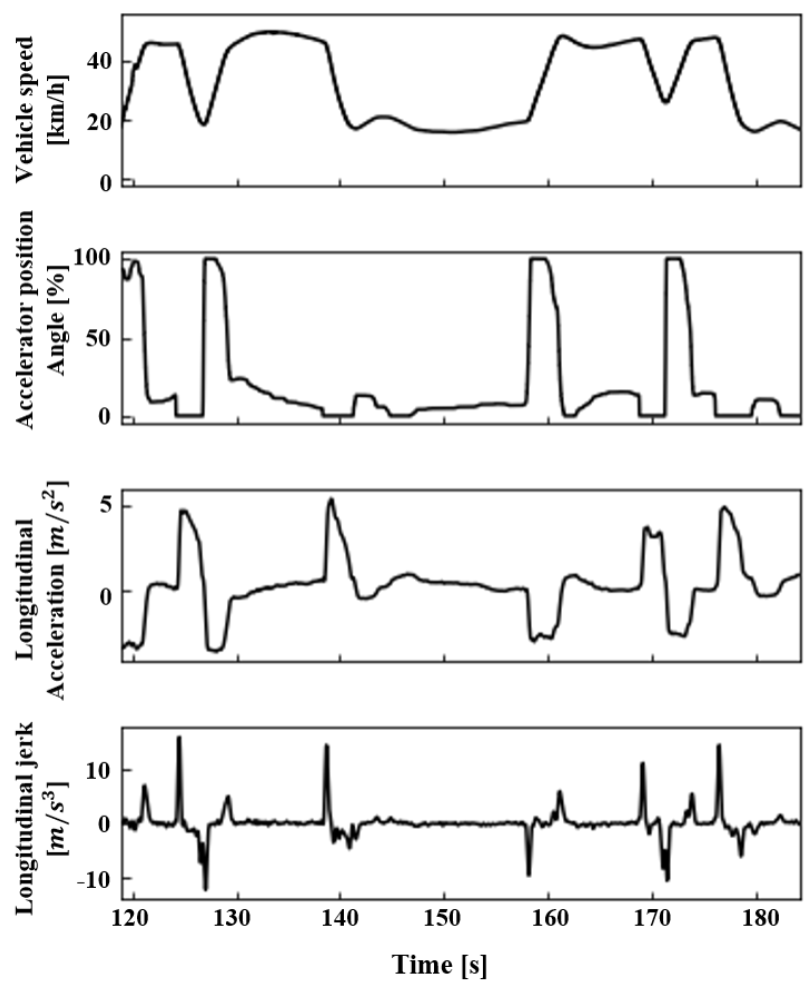

Fig. 3 An example of vehicle behavior indices in T period.

$$
\overline{\text { Jerk }}=\frac{\sum_{i=1}^{n} \int \operatorname{jerk}_{\mathrm{T}_{i}}(t) d t}{n}
$$

The time-series data of SPL and HUM were subtracted by the mean values of Rest period, and the maximum change from $\mathrm{T}_{1}$ period to up to 20 seconds after $T_{n}$ period was extracted after applying a moving average filter.

$$
\begin{aligned}
& \Delta \mathrm{SPL}=\max \left(\mathrm{SPL}(\mathrm{t})-\overline{\mathrm{SPL}_{\mathrm{Rest}}}\right) \\
& \Delta \mathrm{HUM}=\max \left(\mathrm{HUM}(\mathrm{t})-\overline{\mathrm{HUM}_{\text {Rest }}}\right)
\end{aligned}
$$

The time-series data of FHT was subtracted by the mean values of Rest period, and the maximum change from the $\mathrm{T}_{1}$ period to up to 20 seconds was extracted.

$$
\Delta \mathrm{FHT}=\max \left(\mathrm{FHT}(\mathrm{t})-\overline{\mathrm{FHT}_{\mathrm{Rest}}}\right)
$$

R-R intervals (RRI) were extracted from ECG data, and Fast Fourier Transform (FFT) was applied to Rest period and each T periods after cubic spline interpolation at $50 \mathrm{~Hz}$. Then, the low-

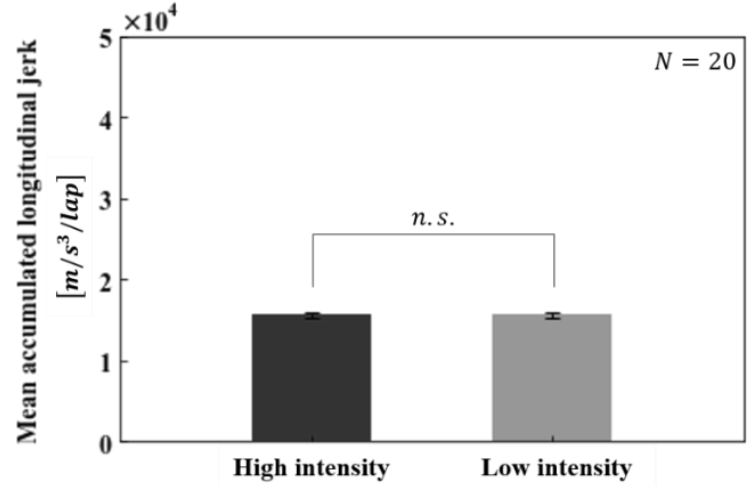

Fig. 4 Mean accumulated longitudinal jerk of vehicle ( $\overline{\text { Jerk }})$.

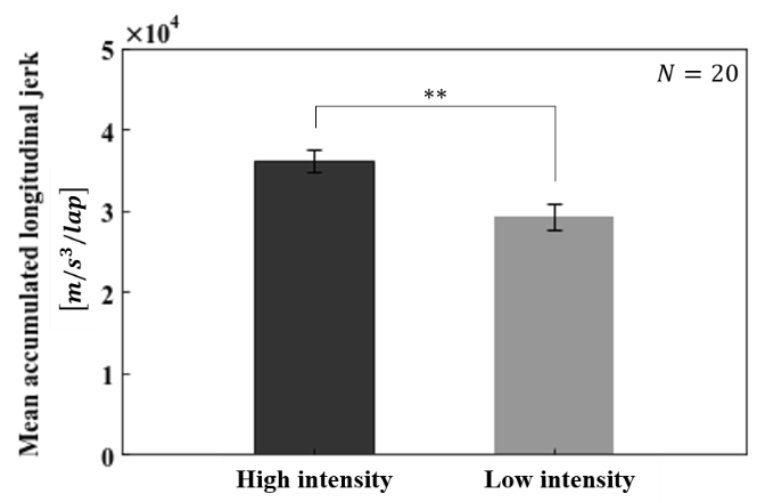

Fig. 5 Mean accumulated longitudinal jerk of head ( $\overline{\text { Jerk }})$.

frequency components (LF) of $0.04 \mathrm{~Hz}$ to $0.15 \mathrm{~Hz}$ and the highfrequency components (HF) of $0.15 \mathrm{~Hz}$ to $0.40 \mathrm{~Hz}$ were extracted. The changes of LF and HF from Rest period to $T_{n}$ period were evaluated.

$$
\begin{aligned}
& \Delta \mathrm{LF}=\mathrm{LF}_{\mathrm{T}_{\mathrm{n}}}-\mathrm{LF}_{\text {Rest }} \\
& \Delta \mathrm{HF}=\mathrm{HF}_{\mathrm{T}_{\mathrm{n}}}-\mathrm{HF}_{\text {Rest }}
\end{aligned}
$$

The changes in the subjective intensities of VAS, discomfort and drowsiness, were calculated as follows.

$$
\Delta \mathrm{VAS}=\mathrm{VAS}_{\mathrm{Q}_{\mathrm{n}}}-\mathrm{VAS}_{\mathrm{Q}_{0}}
$$

The Wilcoxon signed rank test was applied to the evaluation indices except LF and HF for statistical testing. The Mann-Whitney $U$-test was applied to LF and HF, except four trials with missing ECG data. The significant differences of the test result were marked in each figure as follows, **: $p<.01, *: p<.05, n$. s.: $p \geq .05$. 
Vol.12, No.2(2021)

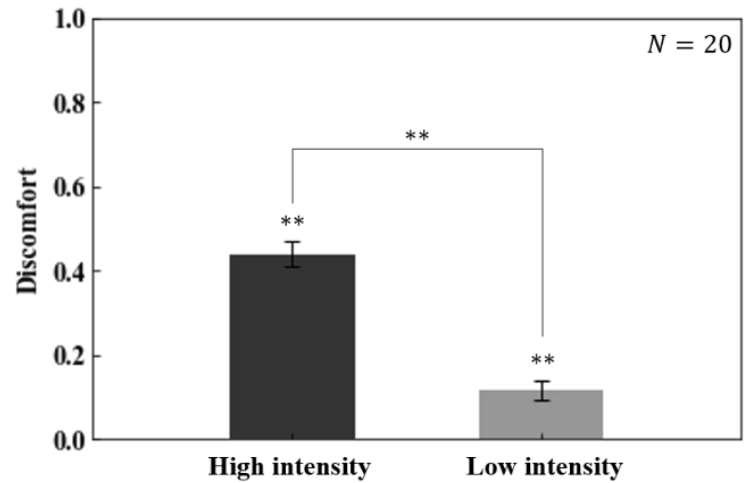

Fig. 6 Change amount of discomfort.

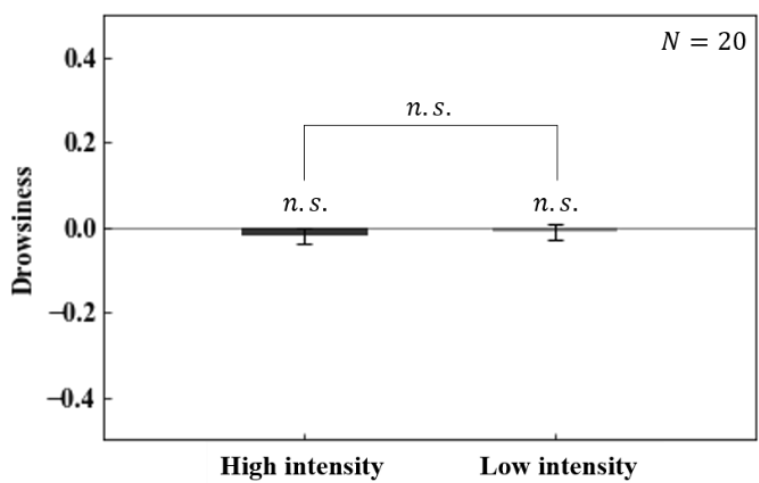

Fig. 7 Change amount of drowsiness.

\section{Results}

\subsection{Condition Classification According to Discomfort Intensity}

Figure 2 shows the change amount of discomfort of each participant. The two different monitor positions induced the different intensities of discomfort. 19 out of 20 participants felt stronger discomfort under the upper position, whereas participant $\mathrm{J}$ felt stronger discomfort under the lower position.

Since the objective of this study is to examine how sweating responds to the different intensities of discomfort, two trials of the upper and lower positions were categorized into two conditions according to discomfort intensity. The condition which the participants felt stronger discomfort was referred to as "high intensity condition", and the other was referred to as "low intensity condition".

\subsection{Experiment Environment}

The mean cabin temperature during the experiments was 24 $\pm 1^{\circ} \mathrm{C}$ for both high- and low-intensity conditions. The cabin temperature during the experiments was in a thermally neutral temperature range ${ }^{(18)}$, and there was no significant difference between the conditions ( $p \geq .05$ ), successfully eliminating the influence of cabin temperature on sweating.

Figure 3 shows the results of the driving information for one of $\mathrm{T}$ periods. Longitudinal acceleration and longitudinal jerk are clearly seen, with the acceleration and deceleration from $20 \mathrm{~km} / \mathrm{h}$ to $50 \mathrm{~km} / \mathrm{h}$ or from $50 \mathrm{~km} / \mathrm{h}$ to $20 \mathrm{~km} / \mathrm{h}$.

Figure 4 shows a comparison of the mean accumulated jerks per $\mathrm{T}$ period between the two conditions, and revealed no significant difference, indicating that the difference between the two conditions was not derived from jerk.

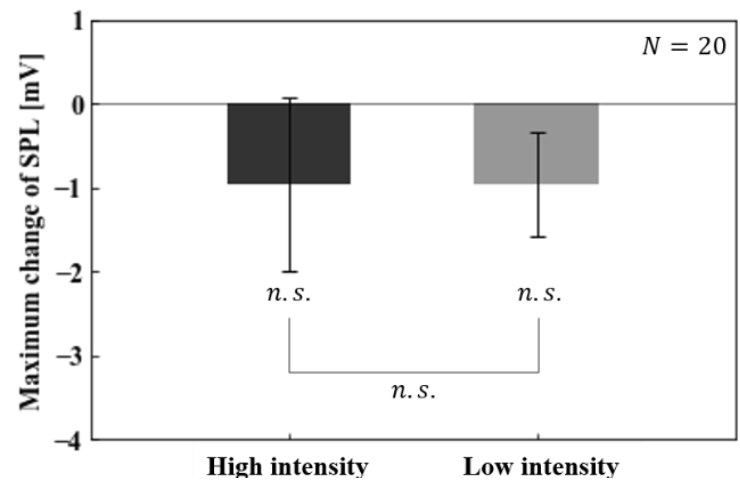

Fig. 8 Maximum change of skin potential level $(\Delta \mathrm{SPL})$.

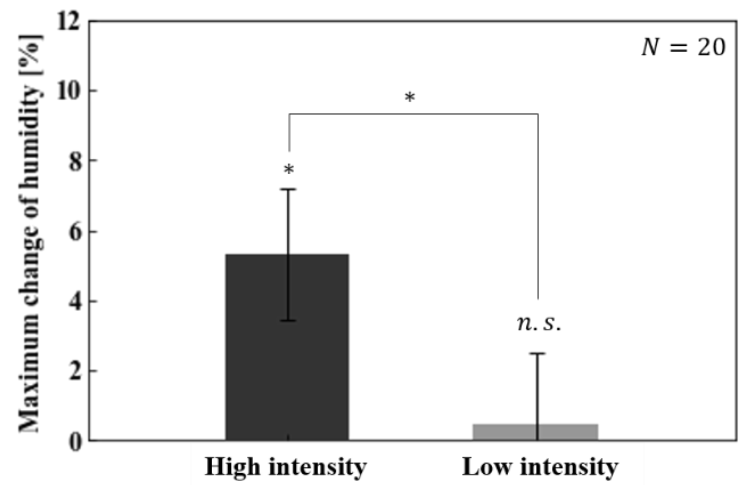

Fig. 9 Maximum change of forehead humidity $(\Delta H U M)$.

\subsection{Head motion}

Figure 5 shows the results of the mean accumulated jerks for head longitudinal movement. The mean accumulated jerks in the longitudinal direction were significantly greater in the high intensity condition than in the low intensity condition $(p<.01)$.

\subsection{Subjective Evaluation}

Figures 6 and 7 shows the change amount of discomfort and drowsiness under the high and low intensity conditions, respectively. Discomfort became significantly stronger after the experiment $(p<.01)$ in both conditions and the high intensity condition induced significantly stronger discomfort than the low intensity condition $(\mathrm{p}<.01)$, while no significant difference was found in drowsiness before and after the start of the experiment or between the conditions ( $p \geq .05$ ).

\subsection{Physiological Responses}

Figure 8 shows the maximum change in SPL, which tended to decrease in both conditions compared to the Rest period. There was no significant difference before and after the start of the experiment, nor was there a significant difference between the two conditions $(p \geq .05)$.

Figure 9 shows the maximum change in HUM. HUM increased significantly in the high intensity condition $(p<.05)$, whereas there was no significant change in the low intensity condition $(p \geq$ $.05)$. The increase was significantly larger in the high intensity condition than the low intensity condition $(p<.05)$.

Figures 10 and 11 shows the changes in LF and HF respectively. LF increased significantly after the start of the experiment in the high intensity condition $(p<.01)$, and the increase was significantly larger in the high intensity condition than in the low intensity condition $(p<.01)$. HF decreased significantly after the start of the experiment in the high intensity condition $(p<.01)$, 
Vol.12, No.2(2021)

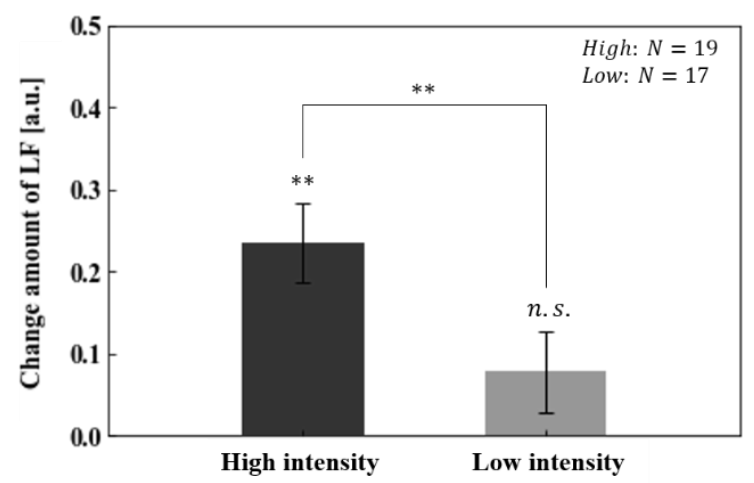

Fig. 10 Change amount of LF $(\Delta L F)$.

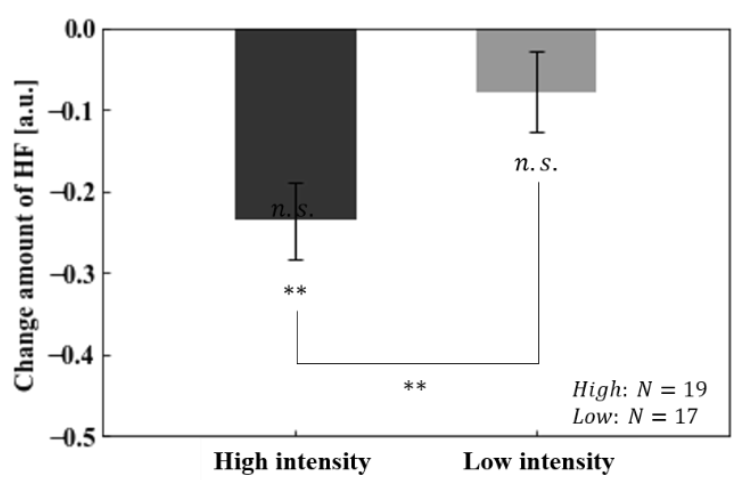

Fig. 11 Change amount of $\operatorname{HF}(\Delta \mathrm{HF})$.

and showed more significant decrease in the high intensity condition than the low intensity condition $(p<.01)$.

Figure 12 shows the maximum change in FHT. There was no significant change in FHT for both conditions, either after the start of the experiment or between the conditions ( $p \geq .05$ ).

\section{Discussion}

\subsection{Differences in Discomfort Between Intensity Conditions}

There was no significant difference in the motion of the vehicle between the two conditions (Figure 4), whereas there was a significant difference in head motion (Figure 5). This difference indicates that the difference of monitor position instead of vehicle motion caused the different head motion. Since head motion increased in the high intensity condition, the authors considered that the discrepancy between the visual information from the display monitor and the vestibular and somatosensory information from the vehicle motion increased in the high intensity condition, inducing stronger discomfort.

\subsection{Physiological Responses Following Discomfort}

Drowsiness affects SPL according to previous studies ${ }^{(19-20)}$, and discomfort associated with MS also caused SPL change as Isu et al. reported ${ }^{(21)}$. As shown in Figure 7, the trials of the two conditions generated no significant changes in drowsiness, which fact indicates the discomfort associated with MS instead of drowsiness caused the changes in SPL. This study showed no significant difference in SPL response between the conditions in spite of the significant difference in discomfort between the conditions. This suggests that SPL response to MS might have large differences among individuals in mild-to-moderate MS.

In contrast, there was more significant increase in HUM for the high intensity condition than the low intensity condition (Figure 9).

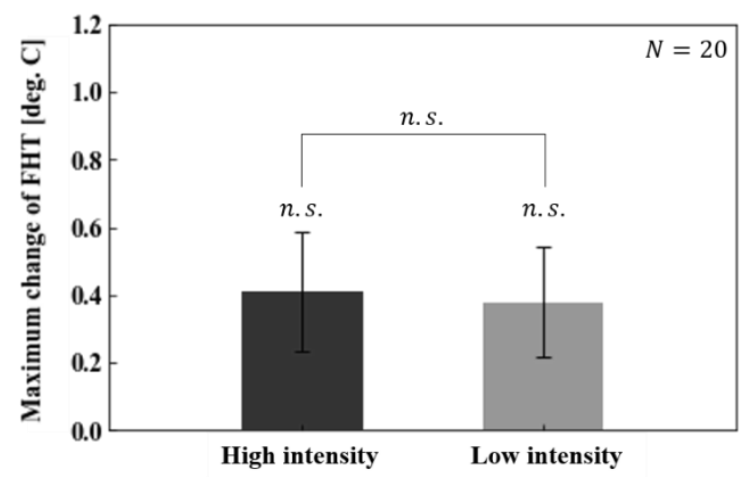

Fig. 12 Change amount of forehead skin temperature ( $\Delta \mathrm{FHT})$.

Table 1 Comparison chart of evaluated indices.

\begin{tabular}{lccc} 
& $\begin{array}{c}\text { Maximum change of } \\
\text { HUM }\end{array}$ & $\begin{array}{c}\text { Maximum change of } \\
\text { SPL }\end{array}$ & $\begin{array}{l}\text { Changes of } \\
\text { HF and LF }\end{array}$ \\
\hline $\begin{array}{l}\text { Conditions of } \\
\text { an evaluated index }\end{array}$ & 0 & $\times$ & \\
$\begin{array}{l}\text { Appearance in } \\
\text { mild-to-moderate MS }\end{array}$ & 0 & $\Delta$ & $\times$ \\
$\begin{array}{l}\text { Specific response of MS } \\
\begin{array}{l}\text { Minor individual } \\
\text { differences }\end{array}\end{array}$ & 0 & $\times$ & 0 \\
$\begin{array}{l}\text { Simplicity of } \\
\text { measurement procedure }\end{array}$ & 0 & $\Delta$ & 0 \\
$\begin{array}{l}\text { Robustness } \\
\text { against vehicle noise }\end{array}$ & 0 & $\Delta$ & 0
\end{tabular}

The cabin temperature of the vehicle was kept at $24 \pm 1^{\circ} \mathrm{C}$ through the experiments, which indicates that discomfort caused sweating, not by the increase of environmental temperature.

LF and HF increased and decreased compared to the start of experiments in the high intensity condition (Figures 10 and 11). As Lin et al. ${ }^{(22)}$ reported, the discomfort induced in the high intensity condition is due to the enhanced activity of sympathetic nervous system.

No significant change was seen in FHT for both conditions during the experiments (Figure 12). According to Nobel ${ }^{(23)}$, MS affects thermoregulatory mechanisms. Cold water exposure normally acts as a vasoconstrictor to prevent deep hypothermia, whereas cold water exposure during experiencing MS weakens vasoconstriction and leads to further deep hypothermia. On the other hand, Kolev et al. ${ }^{(24)}$ reported that MS cause an increase in blood flow to the forehead. These results suggest that inducing more intense MS may lead to an increase in FHT with increased blood flow to the forehead as a result of weakened vasoconstriction.

4.3. Methods for Evaluating Motion Sickness in Real Driving Environment

Table 1 shows the comparison chart of evaluated indices. Although HF and LF differed significantly between the two conditions, it is difficult to evaluate mild-to-moderate MS with HF and LF because they are also a reaction to mental stress and other factors ${ }^{(25-26)}$. On the other hand, the changes of forehead humidity appeared in mild-to-moderate MS because of small individual differences and robustness against vehicle noise. Moreover, the measurement of humidity with a hygrometer is easier than an EDA meter, which needs multiple electrodes.

Under the control of cabin temperature for distinguishing the sweating due to cabin temperature from the sweating due to MS, evaluating MS by changes in forehead humidity, measured with a hygrometer, is effective in real driving environment. 


\section{Shizuka Bando et al / International Journal of Automotive Engineering}

Vol.12, No.2(2021)

\section{Conclusion}

In this paper, the authors focused on sweating. Forehead humidity, which needs only simple procedure, was measured in addition to electrodermal activity used in conventional sweating measurement.

The experiment inducing discomfort of low and high intensity conditions in real driving environment was conducted. The difference between the conditions was not significant in electrodermal activity. On the other hand, humidity above the forehead increased significantly in the high intensity condition and the difference between the conditions was significant in humidity on the forehead. The result indicates small individual differences in response and robustness against vehicle noise.

The evaluation method of mild-to-moderate motion sickness by measuring forehead humidity is effective in real driving environment.

This paper is written based on a proceeding presented at JSAE 2020 Fall Congress.

\section{References}

(1) Strategic Conference for the Advancement of Utilizing Public and Private Sector Data, Strategic Headquarters for the Advanced Information and Telecommunications Network Society: Public-Private ITS Initiative/Roadmap 2019 (2019) (In Japanese).

(2) A. Malokin, G. Circella, P. L. Mokhtarian: How do activities conducted while commuting influence mode choice? Using revealed preference models to inform public transportation advantage and autonomous vehicle scenarios, Transportation Research Part A: Policy and Practice, Vol. 124, pp. 82-114 (2019).

(3) M. Sivak and B. Schoettle: Motion sickness in self-driving vehicles, University of Michigan, Ann Arbor, Transportation Research Institute (2015).

(4) C. Diels and J. E. Bos: Self-driving carsickness, Applied ergonomics, Vol. 53, pp. 374-382 (2016).

(5) S. Joseph, P. Jennings, A. Mouzakitis, and S. Birrell: Too sick to drive: How motion sickness severity impacts human performance. In 2018 21st International Conference on Intelligent Transportation Systems (ITSC), IEEE, pp. 17871793 (2018).

(6) J. Iskander, M. Attia, K. Saleh, D. Nahavandi, A. Abobakr, S. Mohamed, H. Asadi, A. Khosravi, C. P. Lim, and M. Hossny: From car sickness to autonomous car sickness: A review. Transportation research part F: traffic psychology and behaviour, Vol. 62, pp. 716-726 (2019).

(7) International Organization for Standardization: Mechanical vibration and shock -Evaluation of human exposure to wholebody vibration- Part1: General requirements, ISO/DIS 26311.2 (1995).

(8) K. Yasuda: Experimental Studies on the indexes of Motion Sickness and its Autonomic Nervous Symptoms, Practica Otologica, Vol. 11, Supplement 4, pp. 169-188 (1965) (In Japanese).

(9) B. Cheung and K. Hofer: Coriolis-induced cutaneous blood flow increase in the forearm and calf, Brain research bulletin, Vol. 54, No. 6, pp. 609-618 (2001).

(10) Y, Yokota, M. Aoki, K. Mizuta, Y. Ito, and N. Isu: Motion sickness susceptibility associated with visually induced postural instability and cardiac autonomic responses in healthy subjects. Acta oto-laryngologica, Vol. 125, No. 3, pp. 280-285 (2005).
(11) L. T. LaCount, V. Napadow, B. Kuo, K. Park, J. Kim, E. N. Brown, and R. Barbieri: Dynamic cardiovagal response to motion sickness: a point-process heart rate variability study, In 2009 36th Annual Computers in Cardiology Conference (CinC), IEEE, pp. 49-52 (2009).

(12) A. Sjörs, J. Dahlman, T. Ledin, B. Gerdle, and T. Falkmer: Effects of motion sickness on encoding and retrieval performance and on psychophysiological responses, Journal of Ergonomics, Vol. 4, No. 1 (2014).

(13) A. M. Gavgani, K. V. Nesbitt, K. L. Blackmore, and E. Nalivaiko: Profiling subjective symptoms and autonomic changes associated with cybersickness, Autonomic Neuroscience, Vol. 203, pp. 41-50 (2017).

(14) M. Osuga, Y. Nakamura, M. Naito: Toward the Assessment of Passengers' Car Sickness using Physiological Measures, Proceedings of the Fall 2018 Congress of the Society of Automotive Engineers (2018) (In Japanese).

(15) A. Graybiel, C. D. Wood, E. F. Miller II, and D. B. Cramer: Diagnostic criteria for grading the severity of acute motion sickness, Naval Aerospace Medical Institute, Naval Aerospace Medical Center (1968).

(16) P. J. Gianaros, E. R. Muth, J. T. Mordkoff, M. E. Levine, and R. M. Stern: A questionnaire for the assessment of the multiple dimensions of motion sickness, Aviation, space, and environmental medicine, Vol. 72, No. 2, pp. 115-119 (2001).

(17) M. E. McCauley, J. W. Royal, C. D. Wylie, J. F. O'Hanlon, and R. R. Mackie: Motion sickness incidence: Exploratory studies of habituation, pitch and roll, and the refinement of a mathematical mode, Canyon Research Group Inc Goleta Ca Human Factors Research Div. (1976)

(18) ANSI/ASHRAE STANDARD 55-2013: Thermal environmental conditions for human occupancy (2013).

(19) T. Hori, A. Miyashita, and Y. Niimi: Skin potential activities and their regional differences during normal sleep in humans, The Japanese journal of physiology, Vol. 20, No. 6, pp. 657671 (1970).

(20) K, Ishihara, H. Miyata: Physiological Basis on Skin Potential Activity, Humanities review of Kwansei Gakuin University, Vol. 30, No. 3, pp. 75-94 (1980) (In Japanese).

(21) N. Isu, J. Yukio, N. Takahashi: Electrodermal Changes Corresponding to the Degree of Discomfort Induced by Motion Sickness, Technical Report of National Aerospace Laboratory, No. 880, pp. 1-17 (1985) (In Japanese).

(22) C. L. Lin, T. P. Jung, S. W. Chuang, J. R. Duann, C. T. Lin, and T. W. Chiu: Self-adjustments may account for the contradictory correlations between HRV and motion-sickness severity, International journal of psychophysiology, Vol. 87, No. 1, pp. 70-80 (2013).

(23) G. Nobel, A. Tribukait, I. B. Mekjavic, and O. Eiken: dEffects of motion sickness on thermoregulatory responses in a thermoneutral air environment, European journal of applied physiology, Vol. 112, No. 5, pp. 1717-1723 (2012).

(24) O. I. Kolev, C. Möller, G. Nilsson, and L. Tibbling: Responses in skin microcirculation to vestibular stimulation before and during motion sickness, Canadian journal of neurological sciences, Vol. 24, No. 1, pp. 53-57 (1997).

(25) V. Vuksanovicá V. Gal: Heart rate variability in mental stress aloud, Medical engineering \& physics, Vol. 29, No. 3, pp. 344-349 (2007)

(26) Z. Visnovcova, M. Mestanik, M. Javorka, D. Mokra, M. Gala, A. Jurko, A. Calkovska, and I. Tonhajzerova: Complexity and time asymmetry of heart rate variability are altered in acute mental stress, Physiological measurement, Vol. 35, No. 7, pp. 1319-1334 (2014). 